\title{
Hallazgo de Fasciola hepatica (Trematoda: Digenea) en un carpincho (Hydrochaeris hydrochaeris) de la Provincia de Corrientes, Argentina*
}

\author{
Alvarez, J.D. ${ }^{1}$; Moriena, R.A. ${ }^{1}$; Ortiz, M.I. ${ }^{2}$; Racioppi, O. ${ }^{1}$ \\ ${ }^{1}$ Cátedra de Enfermedades Parasitarias, ${ }^{2}$ Cátedra de Genética, Facultad de Ciencias Veterinarias, Universidad \\ Nacional del Nordeste, Sargento Cabral 2139, Corrientes 3400, Argentina. E-mail: parasito@vet.unne.edu.ar.
}

\begin{abstract}
Resumen
Alvarez, J.D.; Moriena, R.A.; Ortiz, M.I.; Racioppi, O.: Hallazgo de Fasciola hepatica (Trematoda: Digenea) en un carpincho (Hydrochaeris hydrochaeris) de la Provincia de Corrientes, Argentina. Rev. vet. 20: 2, 132-134, 2009. El propósito del trabajo fue determinar la prevalencia del trematodo Fasciola hepatica en carpinchos (Hydrochaeris hydrochaeris) de la Provincia de Corrientes (Argentina) y el rol que podría cumplir en la epizootiología de la fasciolosis hepática para otras especies hospedadoras del parásito. Para comprobar la presencia del trematodo se obtuvieron 18 hígados de carpinchos silvestres provenientes de distintas zonas de Corrientes. Del total de órganos examinados, solamente un caso resultó positivo a $F$. hepatica $(5,56 \%)$, identificándose 10 ejemplares por sus caracteres morfológicos y la presencia de abundantes de huevos típicos que se recuperaron durante el examen. Este hallazgo debe considerarse como la primera comunicación del parasitismo de $F$. hepatica en H. hydrochaeris en el país.
\end{abstract}

Palabras clave: Hydrochaeris hydrochaeris, Fasciola hepatica, parasitismo, Corrientes, Argentina.

\begin{abstract}
Alvarez, J.D.; Moriena, R.A.; Ortiz, M.I.; Racioppi, O.: Finding of Fasciola hepatica (Trematoda: Digenea) in a "capybara" (Hydrochaeris hydrochaeris) from Corrientes, Argentina. Rev. vet. 20: 2, 132-134, 2009. The purpose of the study was to determine the prevalence of the fluke Fasciola hepatica in capybaras (Hydrochaeris hydrochaeris) from Corrientes, Argentina, for its possible role in the epizootiology of hepatic fasciolosis in other possible hosts. For this reason, 18 liver samples of wild capybaras were obtained from different areas of Corrientes. For the total of the organs analyzed, only one liver harbored the parasites, identifying 10 specimens according to morphological characteristics and the presence of abundant typical eggs collected during the examination. We consider this finding as the first communication for the country regarding the parasitism by $F$. hepatica on $H$. hydrochaeris.
\end{abstract}

Key words: Hydrochaeris hydrochaeris, Fasciola hepatica, parasitism, Corrientes, Argentina.

\section{INTRODUCCIÓN}

En la Provincia de Corrientes, como en otros lugares de Argentina, Fasciola hepatica es bien conocida por causar una enfermedad parasitaria en los rumiantes, la fasciolosis, responsable de cuantiosas pérdidas económicas de la ganadería ${ }^{14}$. La biología del parásito ha sido extensamente estudiada ${ }^{2,22}$ y hoy se sabe que también afecta a otros animales domésticos y silvestres, además de ser considerada como una zoonosis potencial ${ }^{1}$. En efecto, en Argentina y otros países sudamericanos oca-

*Proyecto 032/06 - SGCyT-UNNE.

Recibido: 23 setiembre 2009 / Aceptado: 27 octubre 2009 sionalmente se registran casos humanos, sobre todo en personas que consumen plantas acuáticas como el berro (Rorippa nasturtium-aquaticum) ${ }^{7,12,17,19}$.

En Corrientes la distribución de $F$. hepatica se ha ampliado en la últimas décadas debido al crecimiento de zonas bajas inundables por causa de embalses artificiales ${ }^{4,15}$ y también por la aparición, en las zonas norte y este de la provincia, de un nuevo huésped intermediario, un pequeño caracol pulmonado denominado Lymnaea columella ${ }^{16,18}$, que se suma a los ya conocidos L. diaphana y L. viatrix localizados en las zonas centro-sur y oeste ${ }^{5,11}$.

Muchos herbívoros silvestres pueden ser hospedadores de $F$. hepatica, entre ellos algunos roedores acuá- 
ticos como el coipo o quiyá (Myocastor coypus) ${ }^{9,13,21}$ y el carpincho (Hydrochaeris hydrochaeris) ${ }^{3,6,8,20}$, los cuales podrían ocupar espacios comunes a huéspedes habituales como los rumiantes domésticos e integrar de esa manera la cadena epizootiológica de la fasciolosis.

Escasos trabajos, la mayoría extranjeros, describen la fasciolosis en roedores acuáticos. La revisión bibliográfica reveló inexistencia de publicaciones que reporten para Argentina el hallazgo de $F$. hepatica en el carpincho, circunstancia que motivó la presente comunicación.

\section{MATERIAL Y MÉTODOS}

En el lapso comprendido entre mayo de 2007 y octubre de 2009 se analizaron 18 hígados de carpinchos silvestres provenientes de distintos departamentos de la Provincia de Corrientes, Argentina, los cuales poseían antecedentes de fasciolosis enzoótica. Las piezas se transportaron congeladas o tratadas con formol al $10 \%$.

En el laboratorio, cada hígado fue disecado con tijera a partir de la vesícula biliar rumbo a los canalículos biliares, para verificar la presencia de especimenes adultos de $F$. hepatica. Posteriormente, el parénquima

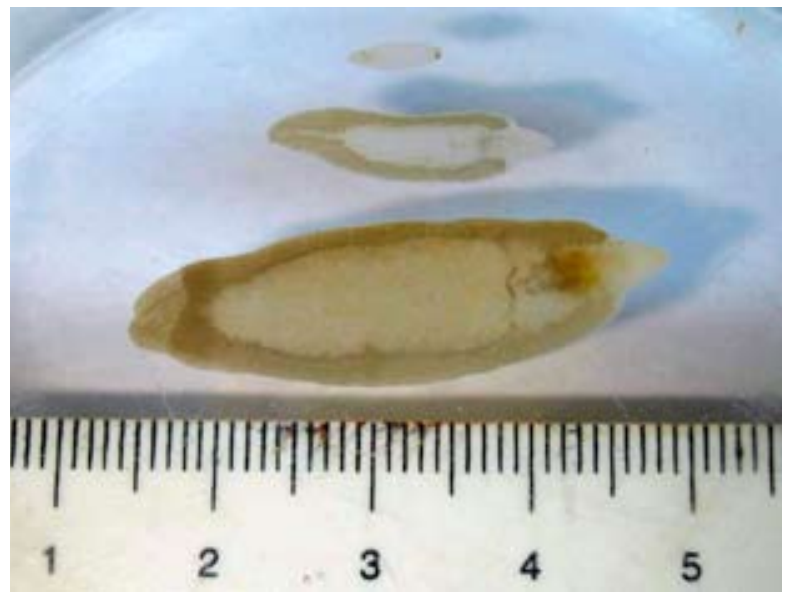

Figura 1. Ejemplares de Fasciola hepatica de diferentes tamaños hallados en carpincho.

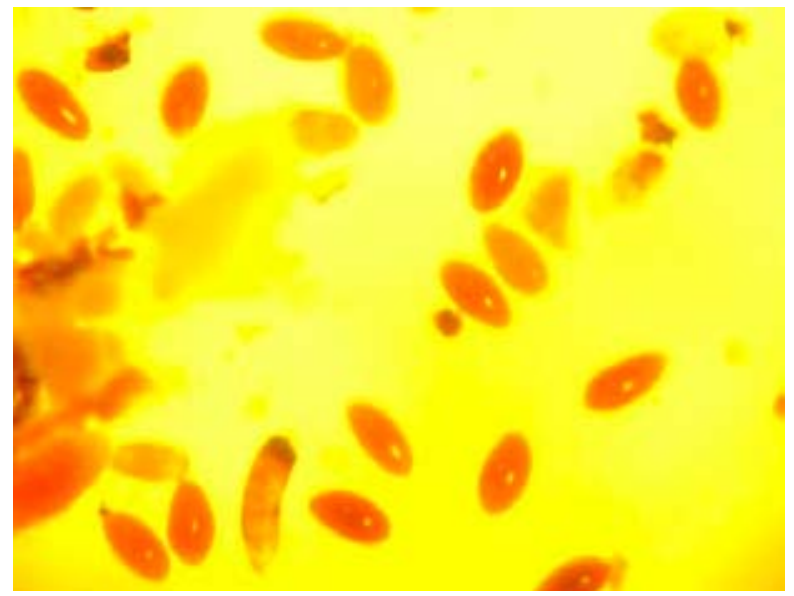

Figura 2. Huevos de Fasciola hepatica coloreados con Lugol (25x). fue seccionado con bisturí o cuchillo en finas lonjas, para poder localizar formas inmaduras del parásito.

Por último, la masa hepática remanente se trituró a mano, se diluyó en agua y se filtró para evidenciar formas inmaduras y huevos. La búsqueda de $F$. hepatica se realizó a simple vista, empleándose medios ópticos para constatar la presencia de huevos. El líquido filtrado se decantó, eliminándose el sobrenadante y aclarando con agua el sedimento, el cual finalmente fue coloreado con Lugol y observado al microscopio.

\section{RESULTADOS}

De los 18 hígados analizados solamente uno $(5,56 \%)$ resultó positivo a $F$. hepatica. Procedía de un animal capturado en Paso Florentín (Departamento General Paz). El órgano presentó aspecto y coloración normales, sin advertirse deformaciones ni fibrosis de los canalículos biliares ni del parénquima hepático.

La identificación del parásito fue realizada de visu, examinando las características morfológicas señaladas por la bibliografía ${ }^{2,22}$. Se hallaron 10 ejemplares del trematodo, cuyas dimensiones variaron entre 6,5 y 35 $\mathrm{mm}$ (Figura 1). El de mayor tamaño fue el único adulto maduro hallado, las demás formas correspondieron a ejemplares inmaduros tempranos e inmaduros tardíos o juveniles.

La observación microscópica reveló la presencia de numerosos huevos de $F$. hepatica intensamente teñidos de color marrón rojizo (Figura 2). La morfología de los huevos, así como sus dimensiones, coincidieron con las descritas para este parásito en la bibliografía. Las medidas oscilaron entre 125 y 150 um (moda 150 um, media aritmética 143,6 um).

\section{DISCUSIÓN}

Este hallazgo debe considerarse como la primera comunicación del parasitismo de $F$. hepatica en $H$. hydrochaeris en Argentina. La exigua cantidad de roedores infestados hallada en este trabajo, torna improbable el papel epizootiológico de los carpinchos como reservorios importantes de la fasciolosis del ganado, por lo menos para el futuro inmediato.

Con respecto a la metodología para evidenciar la presencia de $F$. hepatica en carpinchos, disentimos con investigadores extranjeros que preconizan los resultados positivos obtenidos al emplear técnicas coprológicas $^{3,6}$. Tales identificaciones podrían no ser reales porque $H$. hydrochaeris puede estar parasitado por un trematodo intestinal denominado Taxorchis schistocotyle, cuyos huevos son muy similares al de $F$. hepática, tanto por su forma como por su tamaño ${ }^{10}$. Esta circunstancia podría conducir a la afirmación de "falsos positivos".

Para concluir, hasta que no exista una técnica más precisa para diferenciar entre los huevos de ambos trematodos, se postula que el método más certero es el hallazgo del parásito a través de la necropsia. 


\section{REFERENCIAS}

1. Acha PN, Szyfres B. 1986. Zoonosis y enfermedades transmisibles comunes al hombre y a los animales, $2^{\circ}$ ed., Organización Panamericana de la Salud, Washington, p. 689-696.

2. Andrews SJ. 1999. The life cycle of Fasciola hepatica. In: Fasciolosis (Dalton JP Ed.), CABI Publishing, London, p. $1-29$.

3. Bellato V, Pereira A, Sartor AA, Haubold LP, Centenaro F. 2009. Ocorrencia de Fasciola hepatica na população de capivaras (Hydrochaeris hydrochaeris) e em bovinos (Bos taurus) no município de Timbó, SC. Rev Ciencias Agrovet 8: 66-70.

4. Blanco D, Parera A. 2001. La inundación silenciosa. El aumento de las aguas en los esteros del Iberá: la nueva amenaza de la represa Yacyretá. Ed. Fundación Vida Silvestre Argentina, Buenos Aires, 27 p.

5. Castellanos ZA, Landoni NA. 1981. La familia Lymnaeidae Rafinesque, 1815, en la Argentina. In: Fauna de agua dulce de la República Argentina (Ringuelet RA Ed.), Ed. PROFADU-CONICET, Buenos Aires, p. 55-82.

6. El-Kouba MM, Tietz SM, Pilatti C, Hammann W. 2008. Aspectos gerais da fasciolose e endoparasitoses em capivaras (Hydrochaeris hydrochaeris Linnaeus, 1766) de parques no Paraná, Brasil. Vet em Foco 6: 4-15.

7. Esteban JG, Flores F, Angles R, Mas-Coma S. 1999. High endemicity of human fascioliasis between Lake Titicaca and La Paz valley, Bolivia. Trop Med \& Hyg 93: 151-156.

8. Freyre A, Burges C, Seoane L, Correa I, Rodriguez Piquinela W, Ayala R, Ayala JC, Montanez O. 1979. Parásitos encontrados en autopsias de carpinchos (Hydrochoerus hydrochaeris) en Uruguay. Ann Fac Vet (Uruguay)16: 65-93.

9. Issia L, Pietrokowsky S, Sousa-Figueredo J, Russel Stothard J, Wisnivesky-Colli C. 2009. Fasciola hepatica infection in livestock, guanacos and coypus in two wildlife reserves in Argentina. Vet Parasit 165: 341-344.

10. Lombardero OJ, Moriena RA. 1973. Nuevos helmintos del carpincho (Hidrochoerus hidrochaeris) para la Argentina. Rev Med Vet 54: 265-269.

11. Lombardero O, Moriena R, Racioppi O, Quiroz L. 1979. Bionomía de Limnaea sp. en la Provincia de Corrientes. Rev Vet 2: 5-19.
12. Marcos LA., Maco V, Terashima A, Samalvides F, Miranda E, Tantalean M, Espinoza JR, Gotuzzo E. 2004. Hiperendemicidad de fasciolosis humana en el Valle de Mantaro, Perú: factores de riesgo de la infección por Fasciola hepatica. Rev Gastroenterol (Perú) 24: 158-164.

13. Ménard A, Agoulon A, L'hostis M, Rondelaud D, Collard E, Chauvin A. 2001. Myocastor coypus as a reservoir host of Fasciola hepatica in France. Vet Res 32: 499-508.

14. Moriena RA, Racioppi O, Alvarez JD, Lombardero OJ. 2001. Fasciola hepatica y otros trematodos en bovinos: prevalencia según decomisos en plantas faenadoras en la Provincia de Corrientes (Argentina). Vet Arg 18: 493-498.

15. Moriena RA, Racioppi O, Alvarez JD, Lombardero OJ. 2002. Distomatosis hepática (Fasciola hepatica) y otros trematodos de los rumiantes. Distribución en la Provincia de Corrientes. Reunión de Comunicaciones Cientificas y Tecnológicas, UNNE, Corrientes (Argentina). On line: http://www.unne.edu.ar/cyt/2002/cyt.htm.

16. Moriena RA, Alvarez JD, Pietrokovsky S, Rubel D, Prepeletchi L, Racioppi O, Wisnivesky MC. 2008. Presencia de Pseudosuccinea columella naturalmente infestada con Fasciola hepatica en Santo Tomé (Corrientes, Argentina). Rev Vet 19: 147-149.

17. Pile E, Santos JA, Coelho B, Serra-Freire NM. 2000. Ocorrencia de fascioliase humana no municipio de Volta Redonda, RJ, Brasil. Rev Saude Publ 34: 413-414.

18. Prepelitchi L, Kleiman F, Pietrokosvky S, Moriena RA, Racioppi O, Alvarez JD, Winsnivesky C. 2003. First report of Lymnaea columella Say, 1817 (Pulmonata: Lymnaeidae) naturally infected with Fasciola hepatica (Linaeus, 1758) (Trematoda: Digenea) in Argentina. Mem Inst Oswaldo Cruz 98: 889-891.

19. Rubel D, Prepelitchi L, Kleiman F, Carnevale S, Wisnivesky-Colli C. 2005. Estudio del foco en un caso de fasciolosis humana en Neuquén. Medicina (Buenos Aires) 65: 207-212.

20. Santarem VA, Tostes RA, Alberti H, de Carvalho O. 2006. Fasciola hepatica in capybara. Acta Tropica 98: 311-313.

21. Santos IC, Scaini CJ, Rodrigues AF, Silva Santos IC. 1992. Myocastor coypus as a wild reservoir host of Fasciola hepatica (Linnaeus, 1758). Rev Bras Parasitol Vet 1: 27-30.

22. Skrjabin KI. 1964. Keys to the trematodes of animals and man, University of Illinois Press, Urbana (USA), 351 p. 